\title{
Comparison between Roux-en-Y and Duodenal-jejunal Bypass on hyperglycemia improvements in SD rats
}

\author{
Sen Wang ${ }^{\circledR}$,Wei $\mathrm{Wei}^{\circledR}$,Dong Tang ${ }^{\circledR}$, Nianyuan $\mathrm{Ye}^{\circledR}$, Gang Niu ${ }^{\circledR}$,Daorong Wang ${ }^{\circledR}$, \\ Xiaofang $\operatorname{Sun}^{(3)}$ \\ ${ }^{\circledR}$ The First Clinical Medical College, Nanjing Medical University, Nanjing 210029, People's Republic of China \\ ${ }^{0}$ Department of Gastrointestinal Surgery, Subei People's Hospital of Jiangsu Province (the First Affiliated \\ Hospital of Yang Zhou University), Yangzhou 225001, People's Republic of China \\ ${ }^{8}$ Department of Endocrinology, Subei People's Hospital of Jiangsu Province (the First Affiliated Hospital of \\ Yang Zhou University), Yangzhou 225001, People's Republic of China \\ Correspondence: Daorong Wang, MD, PhD, Department of Gastrointestinal Surgery, Subei People's Hospital of \\ Jiangsu Province, 98 Nantong West Road, Yangzhou, Jiangsu Province, China.
}

\begin{abstract}
Background: To treat Type 2 diabetes mellitus (T2DM), metabolic surgery which Roux-en-Y gastric bypass $(R Y G B)$ and Duodenal-jejunal bypass (DJB) are reported to induce significant improvements on hyperglycemia. In this study we compare the effects of these two surgeries and explore the possible underlying mechanism.

Method: Sprague-Dawley (SD) rats were established of T2DM model and randomly assigned into three groups: the RYGB group, the DJB group and the Control group. Several indexes including body weight, food intake, fasting blood glucose and fasting glucagon-like peptide 1 (GLP-1) were measured during a postoperative 8-week period.

Results : Our results revealed that both RYGB and DJB improved hyperglycemia efficiently along with elevated GLP-1 concentration compared to preoperative period. Moreover, RYGB demonstrated significantly better effects than DJB on decreasing blood glucose and induced a larger increase of GLP-1 levels $(P<0.05)$.

Conclusion: Both RYGB and DJB can significantly decrease blood glucose, RYGB being the more effective one. Postoprative increased GLP-1 levels in varying degrees could be a possible explanation as we seek further into their respective mechanisms.
\end{abstract}

Keywords : Roux-en-Y Gastric Bypass (RYGB). Duodenal-jejunal Bypass (DJB). Type 2 Diabetes Mellitus (T2DM). Glucagon-like Peptide 1 (GLP-1)

\section{Introduction}

The increasing prevalence of Type 2 diabetes mellitus (T2DM) has disturbingly caught people's attention in the twenty-first century. ${ }^{[1][2]}$. Over the years, while surgically treating morbid obese patients with T2DM, doctors were able to observe obvious improvement of diabetic symptoms, thus realizing that surgery could be the cure for T2DM, as barbarian surgery frequently leads to complete resolution of diabetes ${ }^{[3]}$. One of the most favorable procedures is Roux-en-Y gastric bypass surgery. Recently , the duodenal-jejunal bypass has developed as a new approach towards T2DM. Both of them have positive effects on improving hyperglycemia, but their therapeutic mechanisms are yet to be determined.

Roux-en-Y Gastric Bypass (RYGB) has proved effective in controlling diabetic symptoms ${ }^{[1,4-6]}$. Quite a few studies suggest that the improvement can be attributed to the increase of GLP-1, which has multiple synergistic effects on glucose-stimulated insulin secretion ${ }^{[3,7]}$.Duodenal-jejunal bypass (DJB), a more recently applied procedure for T2DM, is also a potential alternative treatment for T2DM, especially those unresponsive to other modalities ${ }^{[8]}$. In the discussion of mechanism, GLP-1 also turn out to play a key role. There are studies indicating that DJB improves $\beta$-cell function and prevents the aggravation of hyperglycemia ${ }^{[3,9]}$. Other hypothesis include the increasing expression of liver glucose transporter protein and liver glucokinase (GCK), leading to poor appetite ${ }^{[10]}$, and the reduction of proinflammatory adipokines and the increase of anti-inflammaroty adipokines, contributing to the improvement of insulin sensitivity ${ }^{[11]}$.

Two hypothesis to explain the effect of RYGB as well as DJB are most widely accepted, the "hindgut hypothesis" and the "foregut hypothesis". The former states that the improvement results from the delivery of undigested food to the distal intestine, amplifying a physiologic signal that enhances glucose metabolism. The latter holds that the positive effect on diabetes should be attributed to the exclusion of the duodenum and proximal jejunum, preventing a pupative signal that aggravates insulin resistance and T2DM. We will further 
discuss these two hypotheses in our study. Under this circumstance, by comparing the surgical validity between those two procedures, we endeavor to explore their respective mechanisms based on the result.

\section{Materials And Methods}

Animals

Thirty male Sprague-Dawley (SD) rats at the age of 7 weeks weighing 260-300g were purchased from Yang Zhou University ,as we followed these studies $^{[4,5,11,14,16]}$ to utilize SD rats as our subjects. SD rats comparatively gain more weights than Wistar and GK rats especially in cases of operating surgeries. Additionally, due to its strong resistance to diseases, SD rats became rather adaptive to variable environments in order to minimize the death rates of rats after surgeries. Moreover, SD rats are much more easily accessible in Yangzhou University and we did set T2DM models on SD rats successfully before, for both of which contributed a lot to its being used as subjects. They were housed individually in cages at a temperature of $21-23^{\circ} \mathrm{C}$ with a $12 \mathrm{~h}$ light-dark cycle (lights on at 07:00, off at 19:00). Rats had free access to water and two-choice chow, consisting of regular chow and high-fat chow (Huabukang Biotech Company, Beijing, China), for 4 weeks ${ }^{[11]}$. Then each rat received an intraperitoneal injection, a middle dose $(40 \mathrm{mg} / \mathrm{kg})$ of STZ (Sigma-Aldrich, St Louis, MO, USA), to destroy part of $\beta$-cells in order to induce hyperglycemia. Rat blood glucose was measured via a portable glucose meter (Bayer, Germany) from the tail vein ${ }^{[1]}$. Rats with blood glucose $\geq 16.7 \mathrm{mmol} / \mathrm{L}$ stably for 2 weeks were considered diabetic ${ }^{[6,11]}$. At the end of the whole process, twenty-seven rats were successfully induced diabetic, while the rest three died with ulcers on the surface of their skin. The study was approved by the Animal Care and Utilization Committee of Yang Zhou University

\section{Experiment design}

Twenty-seven rats were randomly divided into three groups: the Roux-en-Y group (RYGB, $n=9$ ), the Duodenal-jejunal bypass group (DJB, $n=9)$ and the Control group $(, n=9)$. No significant differences were found in terms of weight, blood glucose and food-intake. After 12 hours of fasting and 4 hours of water deprivation, each rat was anesthetized with an intraperitoneal injection of $1 \%$ pentobarbital sodium $(30 \mathrm{mg} / \mathrm{kg})$, followed by an antibiotic (Cefazolin, $50 \mathrm{mg} / \mathrm{kg})^{[12]}$ to prevent potential postoperative infections. Metabolic surgeries were performed, after which we closely observed changes occurring to these rats, including body weight, food intake, fasting blood glucose and GLP-1 levels. During the following 8 weeks, differences in results may give us a clue as to the mechanisms of RYGB and DJB.

\section{Procedures \\ Roux-en-Y}

A midline abdominal $2.5 \mathrm{~cm}$ incision was made and the stomach was divided into two gastric cavities. In human patients, RYGB often bypasses $95 \%$ of the stomach. But according to Xueli Zhang et al ${ }^{[13]}$, because of some anatomic factors and difficulties in stapling, in rats RYGB bypasses only $80 \%$ of the stomach and the rest $20 \%$ gastric pouch was made using a $45 \mathrm{~mm}$ GIA stapler (ETS-Flex Ethicon Endosurgery, Blue Ash, OH, USA). After the small intestine was cut off $8 \mathrm{~cm}$ from the ligament of Treitz, the smaller pouch was connected to distal jejunum with an end-to-side anastomosis to form an alimentary 'Roux' limb. The proximal end of the distal stomach was anastomosed so that a biliopancreatic limb can be made. This limb was reconnected to the jejunum with an end-to-side anastomosis $10 \mathrm{~cm}$ beneath the gastrojejunal anatomosis mentioned, forming "Y" with the alimentary one (Roux-en-Y reconstruction). The remaining intestine formed the common channel as normal. The abdominal wall and skin were closed using 3-0 silk and 5-0 nylon suture ${ }^{[14-15]}$.

\section{Duodenal-jejunal Bypass}

This procedure shared something common with RYGB, yet essentially differed as it kept the stomach intact. First, the same incision was made. Then duodenum was transected $2 \mathrm{~mm}$ distal to pylorus, which, according to Han et $\mathrm{al}^{[8]}{ }^{[8]}$ was critical to operation success rate. Jejunum was also cut $8 \mathrm{~cm}$ from ligament of Treitz and the distal part was connected to pylorus with an end-to-end anastomosis using 7-0 silk suture. The proximal limb was reconnected to jejunum $10 \mathrm{~cm}$ distal to the gastrojejunal anastomosis with an end-to-side suture, using the same silk.

\section{Control}

Nine rats served as diabetic control. They were housed individually and fed with lab chow, upon which no operations were performed. 


\section{Measurement}

Fasting blood glucose was measured after 12 hours of food deprivation, preoperatively and 1, 2, 4, 6 and 8 weeks postoperatively using a glucose meter (Bayer, Germany). At the exact same time, blood samples were transferred and centrifuged. The plasma samples we obtained were stored at $20^{\circ} \mathrm{C}$ until hormone assays. Weight and food intake were monitored weekly after surgery. In particular, food intake on the second day postoperative was noted to observe rats eating behavior after invasive damage. Food intake rate was calculated by the following equation: food intake rate=daily food consumption $(\mathrm{g}) /$ rat body weight $(\mathrm{kg})[8]$. Fasting plasma GLP-1 levels were assayed with an ELISA kit used strictly by the manufacturer's instructions. The blood samples were those we stored as earlier mentioned. The GLP-1 ELISA detected only the active form of GLP-1 with no cross reaction of inactive ones

\section{Postoperative care}

After $24 \mathrm{~h}$ of healing, rats were given chocolate Ensure and water from separate spouts ad libitum for 3 days $^{[16]}$, then a mixture of chocolate Ensure and normal chow. After 1 week, rats started to get accustomed to normal lab chow and food intake grew for the rest recovering days of the experiment. Throughout the postoperative period, rats in all 3 groups were monitored carefully in terms of general conditions, food intake, urine output, blood glucose, etc. Originally a total of 18 rats received surgeries but 5 of them died postoperatively due to infection and intestinal obstruction, 3 from the RYGB group in the first 48 hours and 2 from the DJB group on the second and third day after surgery. In the end, 13 rats made through our experiment.

\section{Statistical Analysis}

All data were analyzed using SPSS 17.0 software and were expressed as mean \pm SD. Differences in groups were analyzed by two-way analysis of covariance. Group results were considered significantly different when $\mathrm{P}<0.05$.

\section{Weight}

\section{Results}

Rats were weighed and recorded weekly. In DJB group, weight dropped moderately from $279.7 \pm 14.1$ $\mathrm{g}$ to $258.4 \pm 12.4 \mathrm{~g}$ in first week, then kept on the rise during the next 5 weeks until reaching peak. No differences were manifested between preoperative and postoperative weight since the fourth week. In the RYGB group, weight dropped sharply from $282.3 \pm 15.5 \mathrm{~g}$ to $240.1 \pm 14.0 \mathrm{~g}$ in the first week, then remained descending in a low rate, in contrast to DJB rats, eventually bottomed at $237.8 \pm 13.6 \mathrm{~g}$. The two surgical procedures presented statistically significant different effects $(\mathrm{P}<0.05)$ on weight control, of which RYGB led to more weight reduction as a result from the exclusion of stomach. In the Control group, weight of rats demonstrated a steadily growing trend and, significantly higher than that of the other groups.

\section{Food intake}

Food consumption rates were both suppressed in two surgery groups in the first 2 days and then took an upward turn. Rats in DJB group took more chow significantly $(\mathrm{P}<0.05)$ than RYGB group during the whole 8 -week period. At the end of the eighth week, food consumption of the RYGB group came to $56.5 \pm 6.6 \mathrm{~g} / \mathrm{kg} / \mathrm{d}$ while that in DJB group came to $78.5 \pm 5.3 \mathrm{~g} / \mathrm{kg} / \mathrm{d}$, which is almost the same as preoperative level. Such an outcome indicated that RYGB had better efficacy in food intake limitation, which might be explained by the different extents of stomach exclusion. In Control group, food intake level remained unchanged for the whole time.

\section{Fasting plasma glucose}

Observation of fasting blood glucose levels of two surgery groups showed both procedures had strong effects on controlling hyperglycemia, while RYGB presented greater influence than DJB significantly $(\mathrm{P}<0.05)$ since postoperative 4 weeks. In the first week, fasting blood glucose concentrations dropped notably from $17.7 \pm 2.3 \mathrm{mmol} / \mathrm{L}$ to $7.8 \pm 1.5 \mathrm{mmol} / \mathrm{L}$ in $\mathrm{RYGB}$ group and from $18.1 \pm 2.1 \mathrm{mmol} / \mathrm{L}$ to $10.6 \pm 1.8 \mathrm{mmol} / \mathrm{L}$ in $\mathrm{DJB}$ group. Since the second week, fasting blood glucose in both groups still remained declining stably, yet with much lower rates. By the end of our observation, fasting blood glucose concentrations in RYGB and DJB groups came to $6.8 \pm 0.7 \mathrm{mmol} / \mathrm{L}$ and $8.6 \pm 0.9 \mathrm{mmol} / \mathrm{L}$ respectively. In Control group, the concentration remained relatively steady around $18 \mathrm{mmol} / \mathrm{L}$ throughout the total 8 weeks, a much higher level than that of two surgery groups $(\mathrm{P}<0.05)$, suggesting both procedures had strong effects on improving hyperglycemia, comparatively a better outcome being shown in RYGB group. 


\section{Fasting blood GLP-1}

To explore possible mechanisms of RYGB and DJB in controlling T2DM, we assayed fasting blood GLP-1 levels using the plasma samples we obtained and stored as is mentioned above. GLP-1 levels in RYGB and DJB groups both elevated with a stable rate from $6.5 \pm 1.5 \mathrm{pmol} / \mathrm{L}$ to $26.7 \pm 1.2 \mathrm{pmol} / \mathrm{L}$ and $7.1 \pm 1.0$ $\mathrm{pmol} / \mathrm{L}$ to $24.0 \pm 1.5 \mathrm{pmol} / \mathrm{L}$ respectively for 6 weeks and declined mildly since then. Peak levels were approximately 3-4 times of their base levels in both surgery groups and were significantly higher than that of control group, leading us to safely conclude that both surgeries strongly increased GLP-1 levels. No differences in terms of fasting blood GLP-1 concentrations between two groups were found until 6 weeks after surgery. GLP-1 levels in RYGB group became significantly higher than that in DJB group since then, which may be of great importance in explaining the different mechanisms in controlling T2DM.

\section{Discussion}

Type 2 diabetes mellitus has become a severe public health problem throughout the globe with a growing number of people suffering from the disease each year. Bariatric surgeries have proved to be effective not only in treating morbid obese patients but also in improving T2DM. Among all surgical procedures, RYGB is considered as a "gold standard procedure" to treat morbid obesity with T2DM or other metabolic disorders and is most frequently recommended and performed. However, RYGB is still a procedure developed to primarily improve morbid obesity and T2DM secondly, making it not a complete metabolic surgery. Duodenal-jejunal bypass is a comparatively new procedure, designed at first as a modification of RYGB to treat only T2DM without affecting weight. RYGB bypasses more than $95 \%$ of the stomach and the proximal jejunum $^{[13]}$ while DJB preserves the whole stomach, making it a more "pure" metabolic operation. In this study, in order to evaluate their efficacies, we used SD rats to directly compare these two surgery procedures through indexes including weight, food intake, fasting blood glucose and GLP-1 levels. It turned out that RYGB has a better effect in treating hyperglycemia than DJB, along with more weight reduction and a larger GLP-1 increase.

As is presented in Fig.2, RYGB limits gastric volume and reduces food intake significantly resulting from the exclusion of the stomach and the formation of the small pouch. Contrary to RYGB group, DJB preserves a full stomach volume and rats maintained a relatively higher level of food intake. Fig. 1 showed that rats in DJB group regained body weight until it reached its preoperative level at postoperative 4 weeks, suggesting this procedure does not affect weight in accord with its original design purpose. This discovery enlightens us on finding alternative options when non-obese patients with T2DM are in need of therapeutic surgeries.

What interests us most is their underlying mechanisms which can be used to explain the different results between RYGB and DJB on controlling hyperglycemia. Both procedures bypass the duodenum and the proximal jejunum, enhancing the delivery of undigested nutrients to the ileum ${ }^{[13]}$. They only vary in the extent of stomach exclusion, as DJB leaves the stomach intact. These mechanical reconstructions lead to some endocrine response changes as adjustments to the relatively new metabolic environment. Hormones like Ghrelin, GLP-1, Gastric inhibitory polypeptide (GIP), Peptide YY (PYY) and cholecystokinin (CCK) ${ }^{[17]}$ were considered key factors in those metabolic changes. Closely associated with the positive effects of bariatric surgery toward metabolic disorders and monitored in our study, GLP-1 is secreted by intestinal K/L-cells of the distal bowel in response to delivery of nutrients, which plays an important part in stimulating insulin secretions ${ }^{[7]}$ and suppressing glucagon secretions ${ }^{[18]}$. It is also reported that GLP-1 and its long-acting analog exendin-4 (Ex-4) regenerate islet $\beta$-cells and inhibit the apoptosis of $\beta$-cells ${ }^{[15]}$. Observation of GLP-1 in our study demonstrated that both RYGB and DJB stimulate higher secretion level of GLP-1 than preoperative level, due to, according to previous studies, the increase in L-cells number ${ }^{[9,19] .}$ Since RYGB bypasses most of the stomach, it is presumed that undigested food passes the intestine rather rapidly, reaching distal ileum and serving as a strong stimuli to provoke K/L-cells secretion of GLP-1. Endocrine intestinal K/L-cells responds by developing hypertrophy and hyperplasia $^{[19]}$ and secrets more GLP-1 longer after stimuli, making GLP-1 levels remarkably higher than preoperative period. DJB leaves the stomach intact, giving it more similarities with normal anatomic construction. We assume that most but not full function of the stomach is preserved, as the digesting function works better than the RYGB group, but not as well as the Control group. Moreover, stomach emptying becomes faster than normal because of the exclusion of pyloric sphincter, leaving the passing food not fully digested, similar to RYGB. Whereas chymes of DJB group also stimulate distal bowel K/L-cells to secret GLP-1, it is more likely a normal physiological response, causing less stimuli than RYGB. Both procedures involve bypassing the duodenum, so we did not intend to study the same part they share during our effort to explore their difference. This hypothesis may explain significant differences in GLP-1 levels and blood glucose between two surgical procedures. Rubino et $\mathrm{al}^{[20]}$ proposed two widely accepted hypotheses, the "foregut" and the 
"hindgut" hypotheses, to explain the effects of RYGB and DJB on T2DM. The "foregut" hypothesis assumed that the effects on controlling hyperglycemia depend on the exclusion of the duodenum and the proximal jejunum, where presumably some putative hormone or signal is secreted. The exclusion prevents duodenum and proximal jejunum from food stimulating, leading to less secretion of the unknown hormone. The other "hindgut" hypothesis assumed that a physiological signal, which is possibly GLP-1, is secreted in response to nutrients stimuli and causes the decrease in blood glucose. Our assumption is mainly based on "hindgut" hypothesis and experiment results seemed to be supportive of this theory. Some previous studies ${ }^{[6-7,}{ }^{12]}$ demonstrated significantly improved effects in insulin sensitivity after RYGB while some showed otherwise ${ }^{[21]}$. They came to the conclusion that DJB could not improve insulin sensitivity as significantly as RYGB does, which may also contribute to the comparatively moderate effect of DJB on hyperglycemia. We did not monitor insulin sensitivity changes, so we have no evidence to support or deny this theory. Meanwhile, although our result supports the "hindgut" hypothesis, it does not deny "foregut" hypothesis,

We still cannot exclude the possibility that there is a putative hormone secreted in the "foregut" to pose a positive impact on hyperglycemia. Moreover, weight reductions occurred remarkably in the first week in both RYGB and DJB group, which is consistent with the time range of reduction in blood glucose. It can not be excluded that there is a causual relationship between weight reduction and glucose decline, as is mentioned in previous study ${ }^{[3]}$. There have been publications ${ }^{[10-11]}$ reporting weight loss independent improvements on hyperglycemia. The influence of weight loss on glucose level remains controversial and further study is needed.

There are several limitations in the present study. First, Since our research is to focus on GLP-1 and mechanism behind it, we only detect two indexes-blood glucose and GLP-1, which does not include many other influential hormones that may account for the results, as GLP-1 may not be the only candidate signal mediator. Some other hormones like GIP secreted by K/L-cells as well might also play a significant role in the mechanism of both procedures, which needs further study. We did not do any insulin sensitivity test either, such as the hyperinsulinemic-euglycemic clamp technique ${ }^{[3]}$, leading to the result that we can not confirm the results concerning insulin sensitivity as is mentioned ${ }^{[21]}$. Second, Our preparations may need to be modified further. The decision of specificity of SD rats was made following several studies, which is also accessible more easily in Yangzhou University due to its animals center. However, this change may lead to uncertain bias which differs from other studies using conventionally Goto-Kakizaki rats, for its fast growth may counteract the weight-reducing effect of the surgeries. Third, the number of samples included in the experiment was relatively small, so results of our study might be statistically biased. Moreover, since the size of our samples was rather small, we assign no subjects to do sham operations to exclude the effects of anesthesia or invasion of the surgery, which may also differ from the conventional way of assigning a sham-operated group. We shall cover all these shortages in the following rats experiments.

In conclusion, RYGB has a better effect on improving hyperglycemia than DJB thanks to more GLP-1 secretion. DJB has a comparatively moderate effect on controlling blood glucose while barely affecting body weight. Studies involving more subjects should be conducted with more hormones being measured to further explore their mechanisms.

\section{References}

[1]. Yu, H., X. Zheng, and Z. Zhang, Mechanism of Roux-en-Y gastric bypass treatment for type 2 diabetes in rats. J Gastrointest Surg, 2013. 17(6): p. 1073-83.

[2]. Ding, D., et al., [Outcomes after laparoscopic surgery for 219 patients with obesity]. Zhonghua Wei Chang Wai Ke Za Zhi, 2011. 14(2): p. 128-31.

[3]. Klein, S., et al., Moderate effect of duodenal-jejunal bypass surgery on glucose homeostasis in patients with type 2 diabetes. Obesity (Silver Spring), 2012. 20(6): p. 1266-72.

[4]. Dailey, M.J., A.A. Moghadam, and T.H. Moran, Jejunal linoleic acid infusions require GLP-1 receptor signaling to inhibit food intake: implications for the effectiveness of Roux-en-Y gastric bypass. Am J Physiol Endocrinol Metab, 2011. 301(6): p. E1184-90.

[5]. Mathes, C.M., et al., Roux-en-Y gastric bypass in rats increases sucrose taste-related motivated behavior independent of pharmacological GLP-1-receptor modulation. Am J Physiol Regul Integr Comp Physiol, 2012. 302(6): p. R751-67.

[6]. He, B., et al., Amelioration in hepatic insulin sensitivity by reduced hepatic lipid accumulation at short-term after Roux-en-Y gastric bypass surgery in type 2 diabetic rats. Obes Surg, 2013. 23(12): p. 2033-41.

[7]. Liu, Y., et al., Roux-en-Y gastric bypass-induced improvement of glucose tolerance and insulin resistance in type 2 diabetic rats are mediated by glucagon-like peptide-1. Obes Surg, 2011. 21(9): p. 1424-31.

[8]. Han, L.O., et al., Key details of the duodenal-jejunal bypass in type 2 diabetes mellitus rats. World J Gastroenterol, 2011. 17(45): p. 5021-7.

[9]. Speck, M., et al., Duodenal-jejunal bypass protects GK rats from \{beta $\}$-cell loss and aggravation of hyperglycemia and increases 
enteroendocrine cells coexpressing GIP and GLP-1. Am J Physiol Endocrinol Metab, 2011. 300(5): p. E923-32.

[10]. Donglei, Z., et al., Effects and mechanism of duodenal-jejunal bypass and sleeve gastrectomy on GLUT2 and glucokinase in diabetic Goto-Kakizaki rats. Eur J Med Res, 2012. 17: p. 15.

[11]. Hu, C., et al., Duodenal-jejunal bypass improves glucose metabolism and adipokine expression independently of weight loss in a diabetic rat model. Obes Surg, 2013. 23(9): p. 1436-44.

[12]. Shin, A.C., et al., Meal-induced hormone responses in a rat model of Roux-en-Y gastric bypass surgery. Endocrinology, 2010. 151(4): p. 1588-97.

[13]. Zhang, X., et al., Effects of Roux-en-Y operations on glucose homeostasis in obese GK rats. Surg Endosc, 2011. 25(11): p. $3493-8$.

[14]. Paranjape, S.A., et al., Improvement in hepatic insulin sensitivity after Roux-en-Y gastric bypass in a rat model of obesity is partially mediated via hypothalamic insulin action. Diabetologia, 2013. 56(9): p. 2055-8.

[15]. Li, Z., et al., Roux-en-Y gastric bypass promotes expression of PDX-1 and regeneration of beta-cells in Goto-Kakizaki rats. World $\mathbf{J}$ Gastroenterol, 2010. 16(18): p. 2244-51.

[16]. Zheng, H., et al., Meal patterns, satiety, and food choice in a rat model of Roux-en-Y gastric bypass surgery. Am J Physiol Regul Integr Comp Physiol, 2009. 297(5): p. R1273-82.

[17]. Mumphrey, M.B., et al., Roux-en-Y gastric bypass surgery increases number but not density of CCK-, GLP-1-, 5-HT-, and neurotensin-expressing enteroendocrine cells in rats. Neurogastroenterol Motil, 2013. 25(1): p. e70-9.

[18]. Shah, M., et al., Contribution of Endogenous Glucagon-Like Peptide 1 to Glucose Metabolism After Roux-en-Y Gastric Bypass. Diabetes, 2014. 63(2): p. 483-93.

[19]. Hansen, C.F., et al., Hypertrophy dependent doubling of L-cells in Roux-en-Y gastric bypass operated rats. PLoS One, 2013. 8(6): p. e65696.

[20]. Rubino, F., et al., The mechanism of diabetes control after gastrointestinal bypass surgery reveals a role of the proximal small intestine in the pathophysiology of type 2 diabetes. Ann Surg, 2006. 244(5): p. 741-9.

[21]. Kindel, T.L., et al., Bypassing the duodenum does not improve insulin resistance associated with diet-induced obesity in rodents. Obesity (Silver Spring), 2011. 19(2): p. 380-7.

Figures: Fig.1 weight:

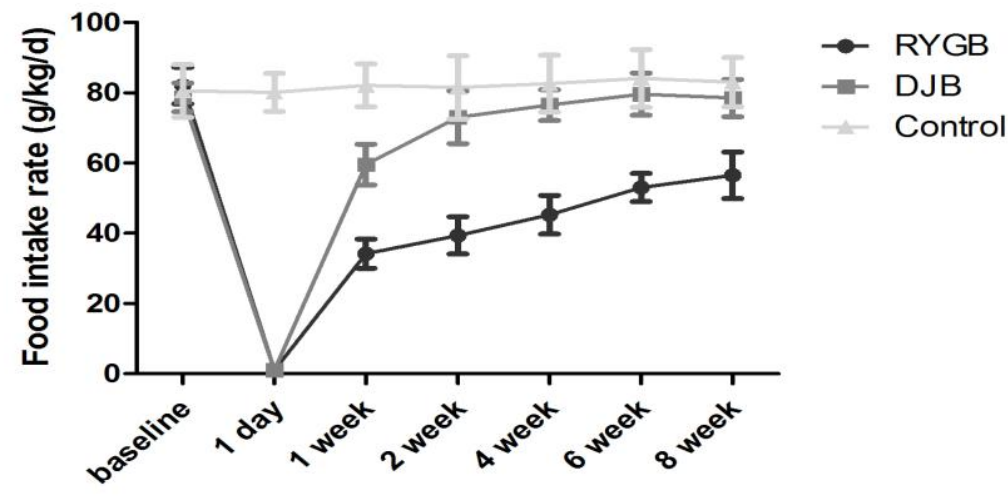

Fig.2 food intake:

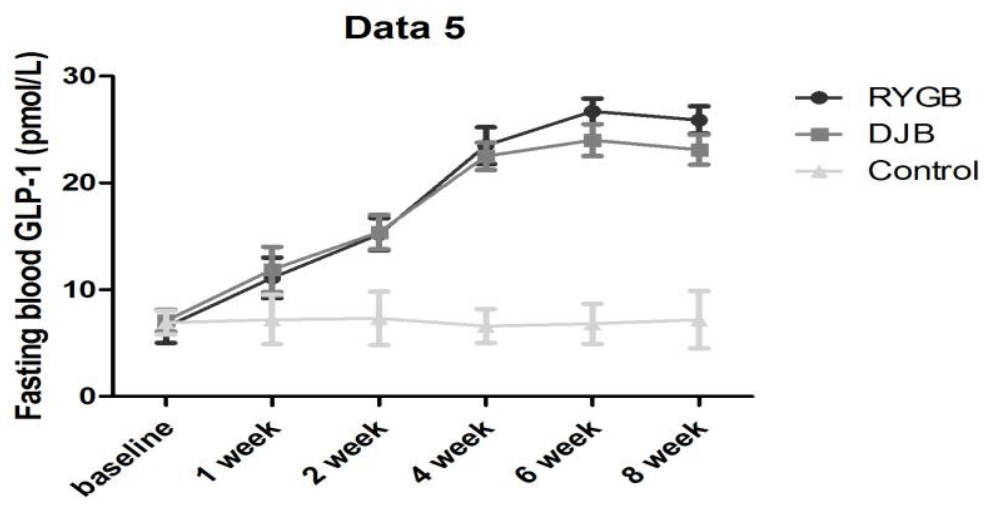


Fig.3 fasting blood glucose:

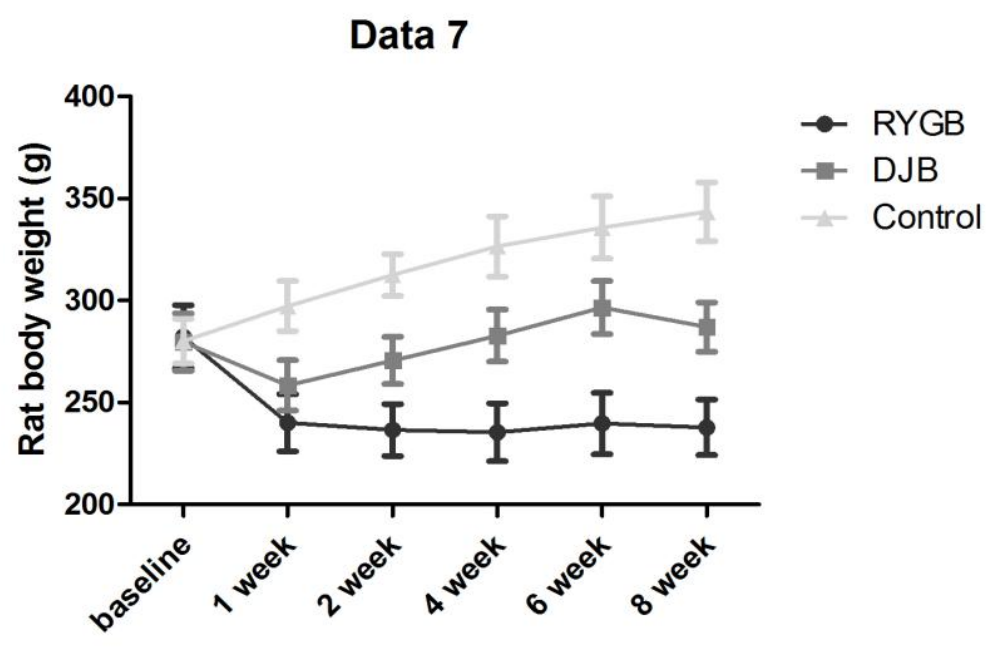

Fig.4 fasting GLP-1:

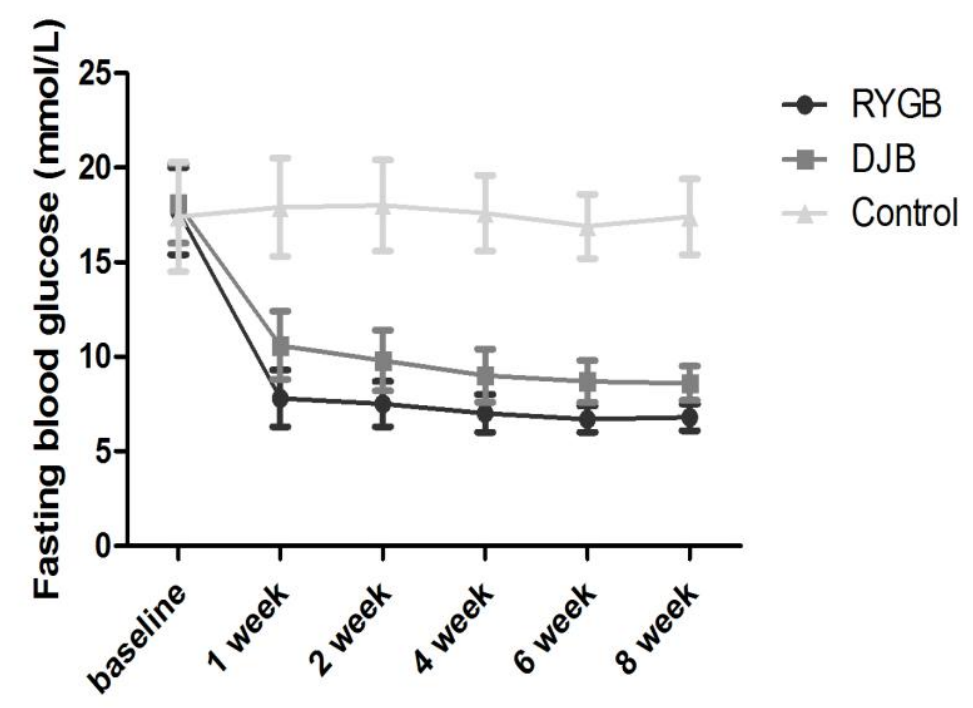

